\title{
Combined fits of the Higgs boson signal at the LHC and the ILC by means of CompHEP
}

\section{E. Boos, V. Bunichev, M. Dubinin}

Skobeltsyn Institute of Nuclear Physics, Moscow State University

E-mail: bunichevatheory.sinp.msu.ru

\begin{abstract}
We analyse the deviations from the standard Higgs phenomenology generated by an effective Lagrangian terms of dimension six. A systematic study of various Higgs boson production channels $(\gamma \gamma, Z Z, W W, b \bar{b}, \tau \bar{\tau})$ at the Large Hadron Collider (LHC) and the International Linear Collider (ILC) in the Standard Model extension by the effective operators is carried out. Statistical methods are used to establish a degree of consistency for the standard Higgs sector with the accumulated LHC and the forthcoming ILC data. Global fits in the two-parametric anomalous coupling space indicating to possible deviations from the standard Higgs-fermion and Higgs-gauge boson couplings are performed.
\end{abstract}

The XXIth International Workshop High Energy Physics and Quantum Field Theory June 23 - June 30, 2013

St.Petersburg, Russia 
The existence of a scalar partcle with properties compatible with the Standard Model (SM) Higgs boson has been reliably established by ATLAS and CMS Collaborations [1]. The signal significance exceeds $7 \sigma$ in both experiments. The properties of the SM Higgs boson could be in principle modified by a new physics at the $\mathrm{TeV}$ scale $\Lambda$, so the question to what extent the observed boson is consistent with the SM Higgs seems the most interesting question to be addressed.

Essential aspects of this problem have been investigated in [2] in a rather general framework of the SM extension by the dimension-six effective operators. At the electroweak energy scale the new physics degrees of freedom can be integrated out and the low-energy effective Lagrangian can be written as

$$
L_{e f f}=L_{S M}+\frac{1}{\Lambda^{2}} \sum_{k=V, F} c_{k \Phi} O_{k \Phi}
$$

where the anomalous couplings $c_{k \Phi}$ modify the SM Higgs boson couplings to the vector bosons and to the fermions. A general set of the dimension-six operators from [2] in the Buchmueller-Wyler basis [3] modified by the subtraction of v.e.v.: $\Phi^{\dagger} \Phi \rightarrow \Phi^{\dagger} \Phi-v^{2} / 2$ (see [4]) to avoid undesirable mixing in the gauge field kinetic terms, can be reduced to a restricted set of only five fermion-Higgs and vector boson-Higgs operators $O_{t \Phi}, O_{b \Phi}, O_{\tau \Phi}$ and $O_{\Phi}^{(1)}, O_{\Phi G}$, disposing the tensor structure of interaction vertices identical to the SM and dependent only on the two anomalous couplings $c_{V}$, which rescales the vector boson-Higgs vertices, and $c_{F}$, which rescales the fermion-antifermionHiggs vertices. The anomalous couplings $C_{n}, C_{m n}$ in front of the dimension-six operators $O_{n}, O_{m n}$ are conformably redefined [2], for example

$$
c_{F}=1+C_{t \Phi} \cdot \frac{v^{2}}{\Lambda^{2}}, \quad c_{V}=1+\frac{v^{2}}{2 \Lambda^{2}} \cdot C_{\Phi}^{(1)}, \quad c_{G}=c_{F}+\frac{6 \pi}{\alpha_{s}} \cdot C_{\Phi G} \cdot \frac{v^{2}}{\Lambda^{2}}, \quad \ldots
$$

$(v=246 \mathrm{GeV})$. The last equation here means that in the case under consideration the one-loop $H \rightarrow \gamma \gamma$ and $H \rightarrow g g$ vertices are "resolved" in the sense that the anomalous parameters $c_{V}$ and $c_{F}$ are included in the one-loop effective $c_{G}$ and $c_{\gamma}$ couplings which parametrise $H \rightarrow g g$ and $H \rightarrow \gamma \gamma$ vertices.

Statistical methods are used to reconstruct the exclusion contours in the anomalous couplings space. In the framework of Bayesian statistics the evidence about the true state of an object under investigation is expressed in terms of degrees of belief (Bayesian probabilities). Baesian inference is based on the assumption $p\left(c_{i} \mid\right.$ data, ESM $) \Rightarrow p\left(\right.$ data $\left.\mid c_{i}, E S M\right) p\left(c_{i} \mid E S M\right)$, where $c_{i}$ is the $n$-parameter set of the SM extension (ESM), the distribution $p\left(c_{i} \mid\right.$ data, ESM) is the posterior probability density function (PDF), $p\left(\right.$ data $\left.\mid c_{i}, E S M\right)$ is the likelihood function defined by the data, and $p\left(c_{i} \mid E S M\right)$ is the proior PDF, representing an apriori degree of belief on $c_{i}$ set. The likelihood $p\left(\right.$ data $\left.\mid c_{i}, E S M\right)$ is defined by a product of likelihoods for individual production channels. Integration of the posterior PDF over a subset $c_{j}(j<n)$ of the $c_{i}$ parameter set we get the inference on the unintegrated parameter(s).

The method of exclusion contours reconstruction [5] introduced to CompHEP version 4 is realized for the one- and the two-dimensional anomalous parameter space. Theoretical signal strength in the infinitely small width approximation (ISW or production $\times$ decay approximation) is defined as

$$
\mu_{i}=\frac{\left[\sum_{j} \sigma_{j \rightarrow h} B r(h \rightarrow i)\right]_{E S M}}{\left[\sum_{j} \sigma_{j \rightarrow h} B r(h \rightarrow i)\right]_{S M}}
$$


where $i$ is the number of Higgs boson decay channel and $j$ is the number of Higgs production process for a given final state. In our extension the efficiences in the numerator and denominator are cancelled. The signal strength can be expressed using the observed cross section $\sigma_{o b s}$, the background cross section $\sigma_{\text {backgr }}$ and the SM signal cross section $\sigma_{\text {signal }}^{S M}$

$$
\hat{\mu}_{i}=\frac{\sigma_{o b s, i}-\sigma_{\text {backgr }, i}}{\sigma_{\text {signal }, i}^{S M}}
$$

The global $\chi^{2}$ for the signal strength is defined as

$$
\chi^{2}\left(\mu_{i}\right)=\sum_{i}^{N_{c h}} \frac{\left(\mu_{i}-\hat{\mu}_{i}\right)^{2}}{\sigma_{i}^{2}}
$$

for the number of production channels $N_{c h}$. Minimization of $\chi^{2} \rightarrow \chi_{\min }^{2}$ gives us $1 \sigma, 2 \sigma$ and $3 \sigma$ regions $\chi^{2}=\chi_{\text {min }}^{2}+\Delta \chi^{2}$ where $\Delta \chi^{2}$ is defined by cumulative distribution function. Assuming that the signal strengths of various channels have Gaussian distributions with the probability density functions having the expected values $\hat{\mu}_{i}$ and the dispersions $\sigma_{i}$ normalized to one, combined PDF for a number of production channels can be found by multiplication of PDF's for individual channels. Combined probability density function is also Gaussian characterized by $\mu_{c}$ and $\sigma_{c}$

$$
\frac{1}{\sigma_{c}^{2}}=\sum_{i}^{N_{c h}} \frac{1}{\sigma_{i}^{2}}, \quad \frac{\hat{\mu}_{c}}{\sigma_{c}^{2}}=\sum_{i}^{N_{c h}} \frac{\hat{\mu}_{i}}{\sigma_{i}^{2}}
$$

The combined PDF allows one to determine, for example, 95\% CL exclusion upper $\mu_{U}$ and lower $\mu_{L}$ limits on the signal strength parameter integrating the combined pdf from $\hat{\mu}$ to $\mu_{U}$ and from $\mu_{L}$ to $\hat{\mu}$, respectively, then equating the result to $0.95 / 2$.

If the SM is fully adequate, the values of $\mu_{i}$ are as close to one as allowed by experimental errors. In the framework of the SM extension by dimension-six effective operators the values of $\mu_{i}$ obviously may depart from one for individual channels, so it is convenient to normalize the signal strengths to the expectation values in the given SM extension $N_{\text {signal }, i, c_{F}, c_{V}, c_{W} \ldots}$ rather than the SM expectation $N_{\text {signal }, i}^{S M}$. In the following fits we are using the signal strength calculated at $m_{H}=125$ $\mathrm{GeV}$. Calculation of the $\Delta \chi^{2}$ for the best fit defines a given number $\times C L$ contours corresponding to the departure of the SM point $(1,1)$ from the best fit point in the $\left(c_{V}, c_{F}\right)$ parameter plane. Contours in the following figures correspond to $65 \%, 90 \%$ and $99 \%$ best fit CL regions with $\Delta \chi^{2}$ less than $2.10,4.61$ and 9.21 , respectively.

At the LHC calculations of complete gauge invariant sets of diagrams are more precise than production $\times$ decay approximation. They take into account (1) untrivial interference between the signal diagrams. The four-lepton final state $l^{+} l^{-} l^{+} l^{-}$, for example, is produced through $H \rightarrow Z^{*} Z^{*}$ and $\gamma \gamma$ intermediate states with interferences not accounted for in the production $\times$ decay approximation; (2) untrivial interference between the signal and the irreducible background diagrams. Although very small for narrow width resonances of the order of a few $\mathrm{MeV}$, the anomalous Higgs boson width can be one order of magnitude greater than the SM total width. Numerical results for complete tree level sets are in some cases very sensitive to Breit-Wigner propagators; (3) lepton and jet distributions, important for detector simulation, are based on correct kinematics when calculated at complete tree level. Correct distributions are oftenly not available for the production $\times$ decay approximation. 
The following Higgs production processes were calculated (1) for the $\gamma \gamma$ event signature: gluon fusion $g g \rightarrow \gamma \gamma$, vector boson fusion (VBF) $q q \rightarrow q q \gamma \gamma$, associated production with vector bosons $q q \rightarrow W \gamma \gamma, q q \rightarrow Z \gamma \gamma$ and the top-antitop quark pair $q q \rightarrow t \bar{t} \gamma \gamma .20$ partonic subprocesses $q \# q \# \rightarrow q \# q \# \gamma \gamma$ were accounted for in the VBF channel, including interference terms between the diagrams. The notation $q \#$ is used to account for all possible combinations of $u, d, c, s$ quarks and anti-quarks; (2) event signatures with four leptons $g g \rightarrow v_{l} \bar{v}_{l} l^{+} l^{-}$and $g g \rightarrow l^{+} l^{-} l^{+} l^{-}$including interference terms between $H \rightarrow W^{+} W^{-}$and $H \rightarrow Z Z$. Vector boson channels $H \rightarrow W^{+} W^{-}$and $H \rightarrow Z Z$ usually mix. In the complete set of six Higgs production diagrams for the channel $p p \rightarrow$ $W W \rightarrow v_{\mu} \bar{v}_{\mu} \mu^{+} \mu^{-}$(WW production via gluon fusion) the $W W^{*}-Z Z^{*}$ interference term is negative with the value of the order of a few percent in comparison with $\left.\left|W W^{*}\right|^{2}+\left|Z Z^{*}\right|^{2}\right)$, cancelling the yield of $\left|Z Z^{*}\right|^{2}$ term. In the $Z Z \rightarrow \mu^{+} \mu^{-} \mu^{+} \mu^{-}$channel the "exchange" interference term $Z Z^{*}-Z Z^{*}$ is positive with the magnitude approximately $20 \%$ of the $\left|Z Z^{*}\right|^{2}$ term. Diagrams with intermediate photons contribute insignificantly in the anomalous coupling space; (3) event signatures with $b \bar{b}$ and $\tau^{+} \tau^{-}$. For $H \rightarrow b \bar{b}$ the processes $q_{1} \bar{q}_{2} \rightarrow W b \bar{b}$ and $q \bar{q} \rightarrow Z b \bar{b}$ were calculated. For $H \rightarrow \tau^{+} \tau^{-}$ channel we calculated $\tau^{+} \tau^{-}, \tau^{+} \tau^{-} \mathrm{VBF}, \tau^{+} \tau^{-} t \bar{t}, \tau^{+} \tau^{-} W$ and $\tau^{+} \tau^{-} Z$ production.

For example, within the ISW for the process $p p \rightarrow v_{\mu} \bar{v}_{\mu} \mu^{+} \mu^{-}$first the $2 \rightarrow 3$ process $p p \rightarrow$ $Z \mu^{+} \mu^{-}$is calculated on a grid $31 \times 31$ in the $\left(c_{V}, c_{F}\right)$ plane, which is then convoluted with the branching $Z \rightarrow \mu^{+} \mu^{-}$calculated on the same grid. A special regime of 'table calculations' (numerical operations with multidimensional tables) has been implemented in CompHEP version 4.5 [6]. Latest data from LC2013 [7] (Hamburg, May 2013) improve significantly the precision which has been achieved for $b \bar{b}$ and $\tau^{+} \tau^{-}$channels in 2012. The contours generated with LC2013 data are shown in Fig.2c. The area at negative $c_{V}$ available with 2012 data, see Fig.2b, disappeared almost completely while contours of the positive $\left(c_{V}, c_{F}\right)$ quadrant demonstrate the consistency of the SM hypothesis with the data on the level of $95 \%$.

It follows from the LHC combined fits that "minimal" extensions of the SM by the dimensionsix $[3,8]$ effective operators, which lead to the rescaling of Higgs couplings, demonstrate insignificant (of the order of a few percent) departures of the nonstandard couplings from their SM limit. Precise measurements are required to study the couplings. Although ATLAS and CMS experiments are sensitive to almost all couplings (except invisible modes and $H \rightarrow c \bar{c}$ ) which have been analysed recently using the LHC data [9], the best sensitivity of individual production channels $g(H \rightarrow P \bar{P}) / g(H \rightarrow P \bar{P})_{S M}-1(P=\gamma, W, Z, \tau, b)$ estimated in a model independent way [10] using the production $\times$ decay approximation is from $5 \%$ to $20 \%{ }^{1}$. Such accuracy will be not sufficient to test the deviations at the percent level, inherent for the MSSM in the decoupling limit or models with composite Higgs boson, where the compositeness scale is of the order of $1 \mathrm{TeV}$.

Clean environment and better signal-to-background ratios of the ILC allows to measure more precisely the couplings of the Higgs boson, which can be observed in all modes including the two-jet decay modes and the invisible modes. Only two production channels are relevant, Higgsstrahlung $e^{+} e^{-} \rightarrow Z H$ and vector boson fusion (VBF) $e^{+} e^{-} \rightarrow v_{e} \bar{v}_{e} H$. Although it will be not possible to measure directly the total width of the scalar which is about $4 \mathrm{MeV}$, it can be indirectly determined separating the events with the Higgs recoiling from the $\mathrm{Z}$ in the Higgsstrahlung process and measuring directly the branching fractions. The accuracies expected from the ILC experi-

\footnotetext{
${ }^{1}$ these numbers are $1 \sigma$ intervals at the energy $14 \mathrm{TeV}$ with the accumulated luminosity $300 \mathrm{fb}^{-1}$, see [11]
} 
ments, estimated from the full detector simulation studies for a realistic accumulated luminosities at $\sqrt{s}=250 \mathrm{GeV}, 500 \mathrm{GeV}$ and $1 \mathrm{TeV}$ can be found in [11]. A combination of simultaneous fits for all possible Higgs boson production channels is presented in Fig.3, where the exclusion contours for $\Delta \chi^{2}=2.10,4.61$ and 9.21 are reconstructed at the energies 250,500 and $1000 \mathrm{GeV}$. Small signal strength error in the channels $e^{+} e^{-} \rightarrow Z H, v \bar{v} H$ with the following $H \rightarrow b \bar{b}$ or $H \rightarrow W W^{*}$ results in the most significant influence of the modes $H \rightarrow b \bar{b}$ and $H \rightarrow W W^{*}$ on the combined $\chi^{2}$ fit. The shape of exclusion contours is most sensitive to the interplay of these two modes, which have opposite behaviour of the cross section dependence $\sigma\left(c_{V}, c_{F}\right)$ on the anomalous couplings. Naive expectation that $H \rightarrow W W^{*}$ channel should be more sensitive to $c_{V}$, than to $c_{F}$, is delusive since the branching ratios are generally speaking not proportional to partial widths because of an untrival dependence of the total width on the anomalous couplings. Despite a small cross section and low expected precision, the channel $H \rightarrow \gamma \gamma$ is very important to exclude the region in the vicinity of $\left(c_{V}, c_{F}\right)=(1,-1)$ which is acceptable for LHC global fits. In the absence of $H \rightarrow \gamma \gamma$ data this region immediately appears for the ILC combined fit due to an asymmetric dependence of $\chi^{2}\left(c_{V}, c_{F}\right)$ on the fermion anomalous coupling $c_{F}$, illustrated by the three-dimensional $\chi^{2}$ in Fig.3a. Asymmetric behaviour with respect to $c_{F}$ is a consequence of asymmetric behavior of $\chi^{2}$ for the $H \rightarrow \gamma \gamma$ channel taken separately. At the energies $\sqrt{s}=500 \mathrm{GeV}$ and $\sqrt{s}=1000 \mathrm{GeV}$ the role of VBF increases with the cross section growth and smaller signal strength error, as illustrated by Fig.3c.

Evaluation of the exclusion contours was carried out for complete gauge invariant sets of diagrams $e^{+} e^{-} \rightarrow(H Z$ or $v \bar{v} H) \rightarrow(f \bar{f} f \bar{f}$ or $f \bar{f} V V)$ with the four-fermion and the two-fermion - two vector boson (including $H \rightarrow g g$ ) final states. Interferences play a role for LHC fits. For the ILC case, some examples also had been found; e.g. in connection with LEP2 analyses it had been mentioned that the four-fermion state $v_{e} \bar{v}_{e} b \bar{b}$ was formed by both $e^{+} e^{-} \rightarrow H Z$ and $e^{+} e^{-} \rightarrow$ $v_{e} \bar{v}_{e} H$ interfering production mechanisms, see details at LEP2 and NLC energies in [12]. Untrivial interferences between signal diagrams, not available in the production $\times$ decay approximation, were found insignificant for our fits, although they could play a role in specific reconstructions of phase space distributions beyond the infinitely small width approximation [13].

\section{Acknowledgements}

The work of E.B., V.B. and M.D. was partially supported by RFBR grant 12-02-93108 and NSh grant 3042.2014.2.

\section{References}

[1] G. Aad et al. (ATLAS Collaboration), Observation of a new particle in the search for the SM Higgs boson with the ATLAS detector at the LHC, Phys. Lett. B716, 1 (2012) (arXiv:1207.7214[hep-ex])

S. Chatrchyan et al. (CMS Collaboration), Observation of a new boson at a mass of $125 \mathrm{GeV}$ with the CMS experiment at the LHC, Phys. Lett. B716, 30 (2012) (arXiv:1207.7235[hep-ex])

[2] E. Boos, V. Bunichev, M. Dubinin, Y. Kurihara, Higgs boson signal at complete tree level in the SM extension by dimension-six operators, Phys. Rev. D89, 035001 (2014) (arXiv:1309.5410 [hep-ph])

E. Boos, V. Bunichev, M. Dubinin, Y. Kurihara, Expectations for the Higgs boson identification at the ILC, SINP MSU report 2014-2-885 (arXiv:1402.4143 [hep-ph]) 
[3] W. Buchmuller, D. Wyler, Effective Lagrangian analysis of new interactins and flavour conservation, Nucl.Phys. B268, 621 (1986)

[4] G. Passarino, NLO inspired effective Lagrangians for Higgs physics, Nucl.Phys. B868, 416 (2013) (arXiv:1209.5538 [hep-ph])

[5] B. Dumont, S. Fichet, G. von Gersdorff, A Bayesian view of the Higgs sector with higher dimensional operators, JHEP 1307, 065 (2013) (arXiv:1304.3369 [hep-ph])

J.R. Espinosa, C. Grojean, M. Muhlleitner, M. Trott, First glimpses at Higgs' face, JHEP 1212, 045 (2012) (arXiv:1207.1717 [hep-ph])

J. R. Espinosa, M. Muhlleitner, C. Grojean, M. Trott, Probing for invisible Higgs decays with global fits, JHEP 1209, 126 (2012) (arXiv:1205.6790 [hep-ph])

J.R. Espinosa, C. Grojean, M. Muhlleitner, M. Trott, Fingerprinting Higgs Suspects at the LHC, JHEP 1205, 097 (2012) (arXiv:1202.3697 [hep-ph])

S. Kraml, B.C. Allanach, M. Mangano, H.B. Prosper, S. Sekmen, C. Balazs, A. Barr, P. Bechtle, G. Belanger, A. Belyaev et al., Searches for new physics: Les Houches recommendations for the presentation of LHC results, Eur.Phys.J. C72, 1976 (2012) (arXiv:1203.2489 [hep-ph])

A. Azatov, R. Contino, J. Galloway, Model-independent bounds on a light Higgs, JHEP 1204, 127 (2012) 127, Erratum-ibid. 1304, 140 (2013) (arXiv:1202.3415 [hep-ph])

[6] E. Boos et al., CompHEP 4.4: automatic computations from Lagrangians to events, Nucl. Instrum. Meth. A534, 250 (2004) (hep-ph/0403113) A. Pukhov et al., CompHEP 3.3 users' manual, hep-ph/9908288;

see also http://theory.npi.msu.su/comphep

[7] D. Zanzi (on behalf of ATLAS collaboration), Latest Higgs results from ATLAS, A. Savin (on behalf of CMS collaboration), Higgs results from CMS, reported on the European Linear Collider Workshop, 27 - 31 May 2013, DESY, Hamburg.

[8] J. Elias-Miro, C. Grojean, R. Gupta, D. Marzocca, Scaling and tuning of EW and Higgs observables, arXiv:1312.2928 [hep-ph], 2013

R. Alonso, E. Jenkins, A. Manohar, M. Trott, Renormalization group evolution of the SM dimension-six operators, arXiv:1312.2014 [hep-ph], 2013

C. Chen, S. Dawson, C. Zhang, Electroweak effective operators and Higgs physics, arXiv:1311.3107 [hep-ph], 2013

I. Brivio, T. Corbett, O.J.P. Eboli, M.B. Gavela, J. Gonzalez-Garcia, L. Merlo, S. Rigolin, Disentangling a dynamical Higgs, arXiv:1311.1823 [hep-ph], 2013

J. Elias-Miro, J.R. Espinosa, E.Masso, A. Pomarol, Higgs windows to new physics through $d=6$ operators, arXiv:1308.1879 [hep-ph], 2013

R. Contino, M. Ghezzi, C. Grojean, M. Muhlleitner, M. Spira, Effective Lagrangian for a light Higgs-like scalar, JHEP 1307, 035 (2013) (arXiv:1303.3876 [hep-ph])

T. Corbett, O.J.P. Eboli, J. Gonzalez-Fraile, M.C. Gonzalez-Garcia, Constraining anomalous Higgs interactions, Phys.Rev. D86, 075013 (2012) (arXiv:1207.1344 [hep-ph])

B. Grzadkowski, M. Iskrzynski, M. Misiak, J. Rosiek, Dimension-six terms in the Standard Model Lagrangian, JHEP 1010, 085 (2010) (arXiv:1008.4884 [hep-ph])

K. Whisnant, J.M. Yang, B.-L. Young, X. Zhang, Dimension six CP-conserving operators of the third family quarks and their effects on collider observables, Phys. Rev. D56, 467 (1997) (hep-ph/9702305) K.Hagiwara, S. Ishihara, R. Szalapski, D. Zeppenfeld, Low-energy effects of new interactions in the electroweak boson sector, Phys. Rev. D48, 2182 (1993) 
[9] CMS Physics Analysis Summaries, Combination of standard model Higgs boson searches and measurements of the properties of the new boson with a mass near $125 \mathrm{GeV}$, CMS-PAS-HIG-13-005, see https: / / cds.cern.ch/record/1542387

ATLAS Collaboration, Measurements of Higgs boson production and couplings in diboson final states with the ATLAS detector at the LHC, arXiv:1307.1427v1 [hep-ex]

[10] M. Peskin, Estimation of LHC and ILC capabilities for precision Higgs boson coupling measurements, arXiv:1312.4974 [hep-ph], 2013

[11] The International Linear Collider Technical Design Report - Volume 2: Physics, ed. by H.Baer, T.Barklow, K.Fujii, Y. Gao, A.Hoang, S.Kanemura, J.List, H.Logan, A.Nomerotski, M.Perelstein, arXiv:1306.6352 [hep-ph], 2013

[12] E. Boos, M. Dubinin, L. Dudko, Higgs boson production under the resonance threshold at LEP-2, Int.J.Mod.Phys. A11, 5015 (1996) (hep-ph/9602220)

E. Boos, M. Sachwitz, H.J. Schreiber, S. Shichanin, The reaction $e^{+} e^{-} \rightarrow v \bar{v} b \bar{b}$ and the Higgs signal at LEP-200 and NLC, Int.J.Mod.Phys. A10, 2067 (1995)

M. Dubinin, V. Edneral, Y. Kurihara, Y. Shimizu, Complete tree level calculation of the process $e^{+} e^{-} \rightarrow v \bar{v} b \bar{b}$ and the Higgs signal at LEP-200 and next linear colliders, Phys.Lett. B329, 379 (1994)

[13] M.Grunewald, G.Passarino et.al., Four fermion production in electron-positron collisions, Reports of the working groups on pecision calculations for LEP2 physics, CERN report 2000-09-A, p. 1-135 (hep-ph/0005309) 
H $2 X$

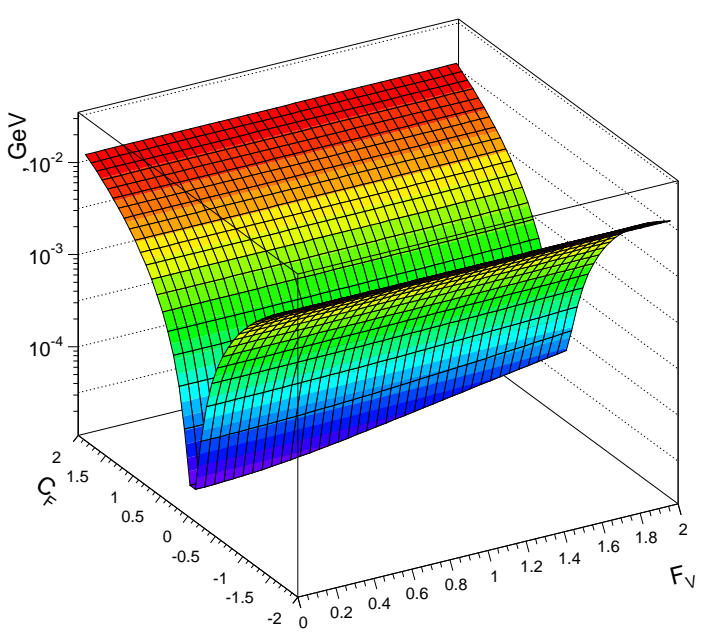

(a)

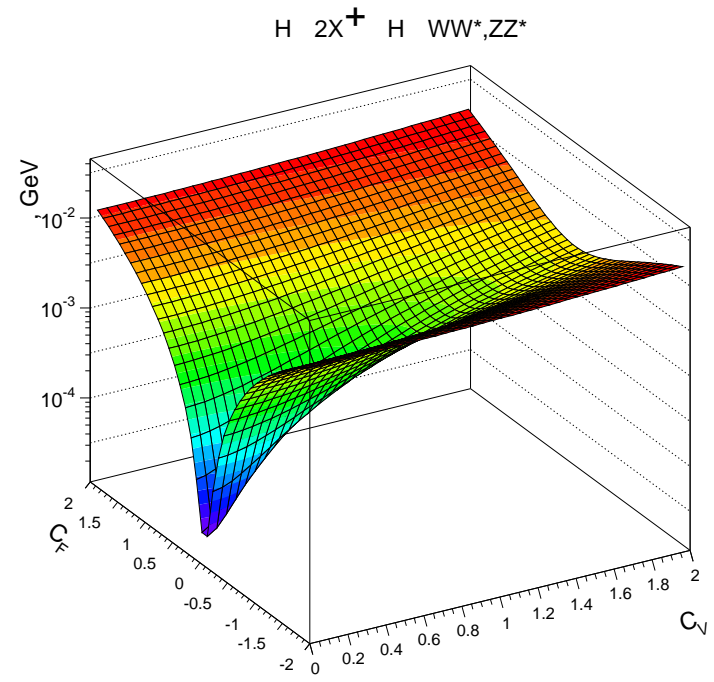

(c)

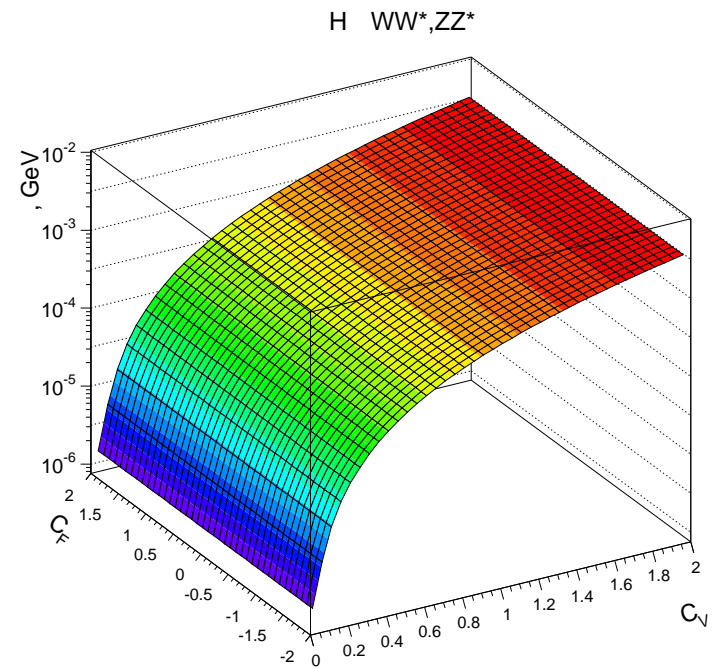

(b)

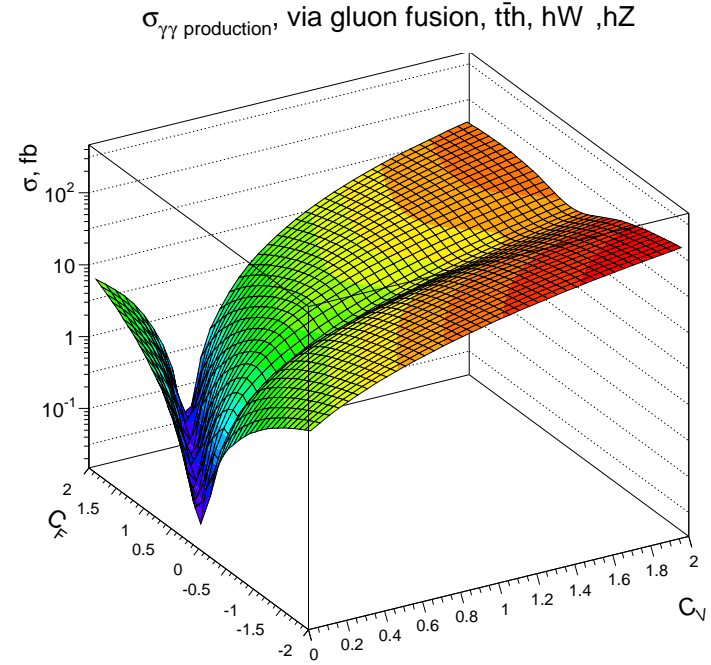

(d)

Figure 1: Three-dimensional plots of the Higgs boson decay width as a function of $\left(c_{V}, c_{F}\right)$ : (a) - two-body decays, (b) - $W W^{*}$ and $Z Z^{*}$ modes only, (c) - total decay width, (d) - total cross section for $\gamma \gamma$ production processes at the LHC, $\sqrt{s}=8 \mathrm{TeV}$. 
$\chi^{2}$, LHC 2012

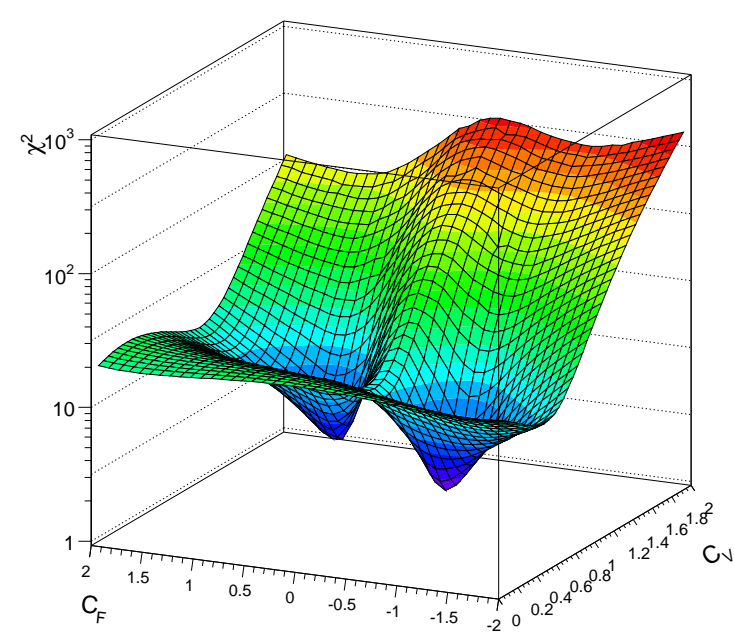

(a)

Global fit (LHC 2013)

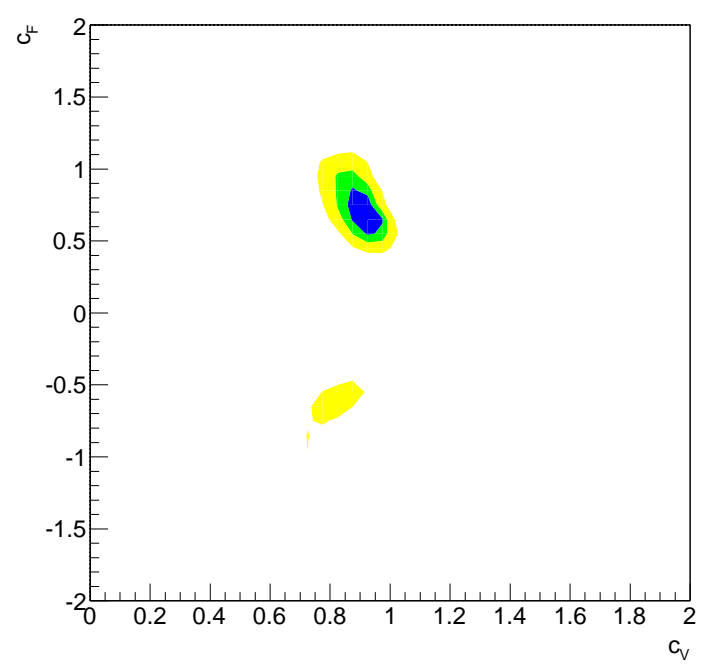

Global fit (LHC 2012)

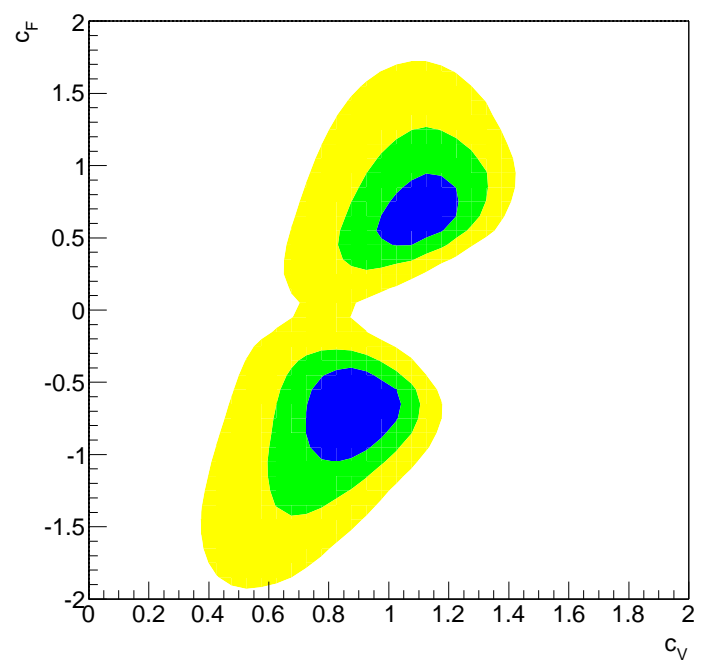

(b)

(c)

Figure 2: (a) Three-dimensional plot of $\chi^{2}$ as a function of $\left(c_{V}, c_{F}\right), \mathrm{LHC} \sqrt{s}=8 \mathrm{TeV}$ - all channels, (b) thre exclusion contours for the combined $\chi^{2}$ fit in the $\left(c_{V}, c_{F}\right)$ plane based on 2012 data, (c) - the same based on 2013 data. Blue, green and yellow areas correspond to $\Delta \chi^{2}=2.10,4.61$ and 9.21 (CL of the fit is $65 \%$, $90 \%$ and $99 \%$ ), respectively. 


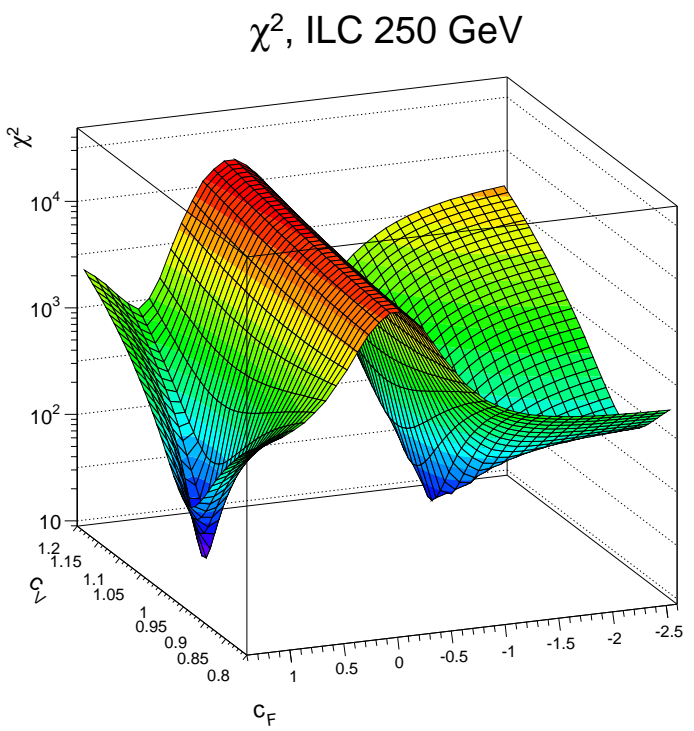

(a)

Global $\chi^{2}$ fit, ILC $500 \mathrm{GeV}$

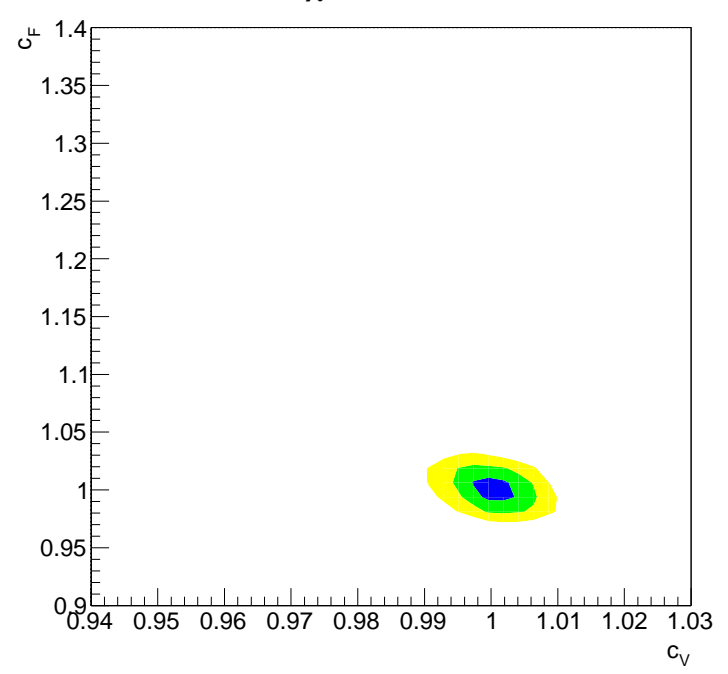

(c)
Global $\chi^{2}$ fit, ILC $250 \mathrm{GeV}$

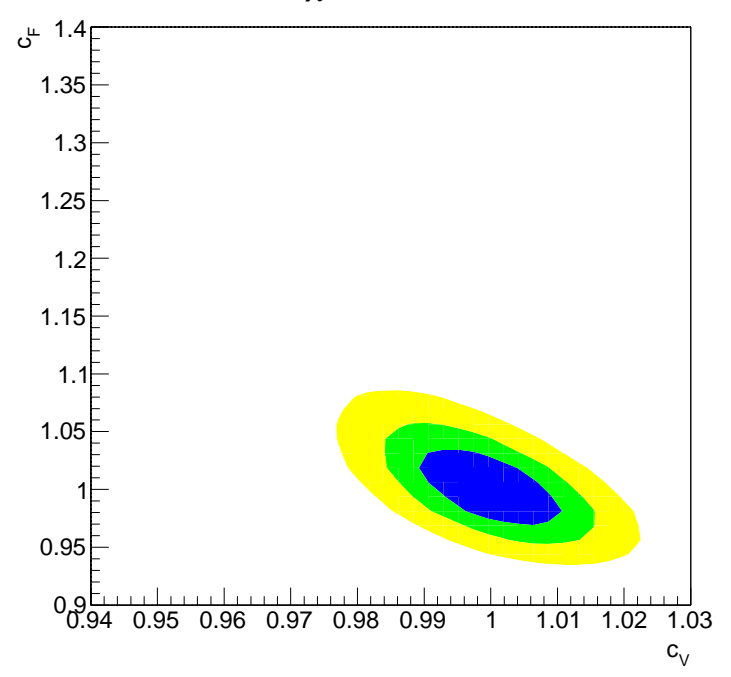

(b)

Global $\chi^{2}$ fit, ILC $1000 \mathrm{GeV}$

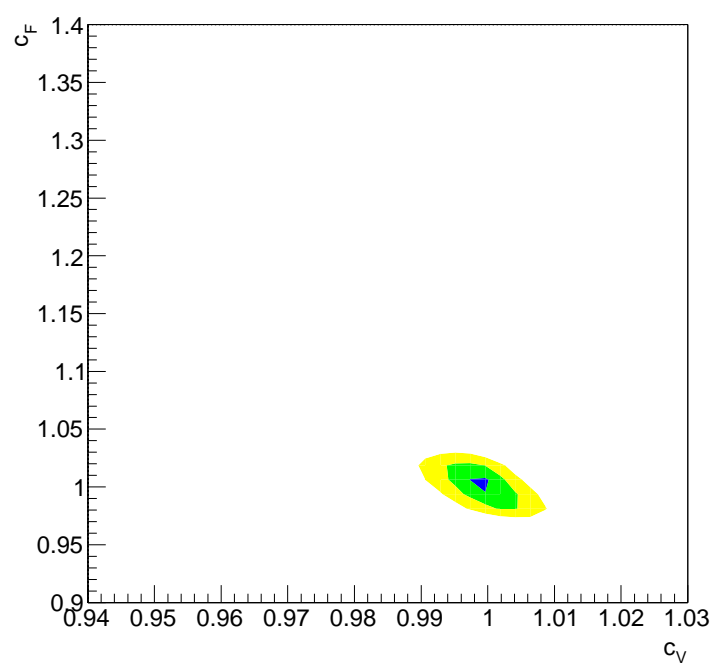

(d)

Figure 3: (a) Three-dimensional plot of $\chi^{2}$ as a function of $\left(c_{V}, c_{F}\right)$ for the ILC, $\sqrt{s}=250 \mathrm{GeV}$, all channels, (b)- exclusion contours for the combined $\chi^{2}$ fit in the $\left(c_{V}, c_{F}\right)$ plane, $\sqrt{s}=250 \mathrm{GeV}$, (c) $-\sqrt{s}=500 \mathrm{GeV}$, (d) $\sqrt{s}=1000 \mathrm{GeV}$. Blue, green and yellow areas correspond to $\Delta \chi^{2}=2.10,4.61$ and 9.21 (CL of the fit is $65 \%$, $90 \%$ and $99 \%$ ), respectively. 